\title{
BMJ Open Primary care professionals' attitudes towards digital health interventions for common mental disorders: study protocol for a mixed methods systematic review
}

Maria Hanf, ${ }^{1}$ Julian Hirt, ${ }^{2,3}$ Marjan van den Akker (D) ${ }^{1,4,5}$

To cite: Hanf M, Hirt J, van den Akker M. Primary care professionals' attitudes towards digital health interventions for common mental disorders: study protocol for a mixed methods systematic review. BMJ Open 2021;11:e045657. doi:10.1136/ bmjopen-2020-045657

- Prepublication history for this paper is available online. To view these files, please visit the journal online (http://dx.doi org/10.1136/bmjopen-2020045657).

Received 26 0ctober 2020 Accepted 14 May 2021
Check for updates

(c) Author(s) (or their employer(s)) 2021. Re-use permitted under CC BY-NC. No commercial re-use. See rights and permissions. Published by BMJ.

For numbered affiliations see end of article.

\section{Correspondence to}

Professor Marjan van den Akker; m.vandenAkker@ allgemeinmedizin.uni-frankfurt. de

\section{ABSTRACT}

Introduction Mental disorders such as depression are common, and an estimated 264 million people are affected by them throughout the world. In recent years, studies on digital health interventions to treat mental disorders have shown evidence of their efficacy, and interest in using them has increased as a result. In the primary care setting, depression and anxiety are the two most frequently diagnosed and treated mental disorders. When they do not refer them to specialists, primary care professionals such as general practitioners treat patients with mental disorders themselves but have insufficient time to treat them adequately. Furthermore, there is a shortage of psychotherapists and those that exist have long waiting lists for an appointment. The purpose of this mixed methods systematic review is to explore the attitudes of primary care professionals towards the use of digital health interventions in the treatment of patients with mental disorders. Their attitudes will provide an indication whether digital mental health interventions can effectively complement standard care in the primary care setting. Methods and analysis We searched for qualitative, quantitative and mixed methods studies published in English, German, Spanish, Russian, French and Dutch after January 2010 for inclusion in the review. The included studies must involve digital mental health interventions conducted via computer and/or mobile devices in the primary care setting. The search was conducted in July 2020 in the following electronic bibliographic databases: MEDLINE, Embase, CINAHL, PsycINFO and Web of Science Core Collection. Two reviewers will independently screen titles, abstracts and full texts and extract data. We will use the 'Integrated methodology' framework to combine both quantitative and qualitative data.

Ethics and dissemination Ethical approval is not required. We will disseminate the results of the mixed methods systematic review in a peer-reviewed journal and scientific conferences.

PROSPERO registration number CRD42020188879.

\section{INTRODUCTION}

Mental disorders such as depression are common, and an estimated 264 million people are affected by them throughout the
Strengths and limitations of this study

- This mixed methods review focuses on the mental disorders that are most frequently treated in primary care and will not cover all mental disorders.

- Strengths of this study are a comprehensive literature search using database search and supplementary search methods.

- The literature search and study selection will be conducted by two reviewers.

- The systematic review will provide a range of quantitative and qualitative data on attitudes towards various types of digital treatment for patients with mental disorders in a primary care setting.

world. ${ }^{12}$ Besides neurological and substance use disorders, major depressive and anxiety disorders constitute a significant proportion of overall global disease burden. ${ }^{3}$ Besides personal suffering, depression is a risk factor for suicide. ${ }^{4}$ Furthermore, ischaemic heart disease can be caused by mental disorders. ${ }^{3}$ In most countries, treatment rates for mental disorders are low and 'health systems worldwide need to respond to this rising burden by implementing proven, cost-effective interventions $[\ldots]]^{3}$. There is a shortage of mental health specialists, especially in rural areas, and studies indicate that psychotherapists and specialists have long waiting lists. ${ }^{5-7}$ Depression and anxiety are the two most frequently diagnosed and treated mental disorders in the primary care setting. ${ }^{57}$ In Germany, onethird of the general population have mental health problems, ${ }^{8}$ and approximately $50 \%$ of German patients with depressive disorders are treated by a primary care professional. ${ }^{9}$ In many western countries, persons with depressive disorders generally present in the primary care setting first, and general practitioners (GPs) therefore play a central role 
as 'gatekeepers' in the treatment of such patients. Sometimes they treat the patients themselves, and at other times they refer them to specialists. ${ }^{7-11}$ But GPs have insufficient time to treat patients with mental health conditions adequately. ${ }^{56}$ One of the main reasons why GPs refer such patients to specialists is that they do not have the appropriate skills to provide optimal care for them. However, patients with mental health problems are often reluctant to accept a referral, ${ }^{5}$ so GPs frequently agree to treat the patients themselves. ${ }^{6}$

Digital technologies (E-health) are continually evolving and an emerging, fast-developing field of research and practice. ${ }^{12}$ Digital treatment options provide the healthcare system with new opportunities ${ }^{13}$ and the increasing use and demand for internet and mobile technologies has led to a shift in traditional ways of delivering healthcare. ${ }^{14}$ The majority of primary care patients $(78 \%, \mathrm{n}=638)$ are internet users. ${ }^{15}$

Digital interventions can take various forms. Examples of these are text-based interventions that can be downloaded onto a smartphone or used on the internet, and that make use of self-help programmes, or interventions in which audiovisual communications such as videos or films are used to provide disease-specific information. Further interventions are interactive and make use of email and text message functions. ${ }^{12} 16$

In recent years, interest in using digital mental health interventions to treat mental disorders as a treatment alternative to traditional non-pharmaceutical therapies (eg, face-to-face therapy) has increased. ${ }^{17-19}$ Digital mental health interventions (E-mental health) involve the use of information and communication technology to improve mental health conditions. ${ }^{20}$ E-mental health is an umbrella term used to describe the application of new media, especially internet-related technologies. E-mental health encompasses the use of digital technologies and new media, ranging from the delivery of screening and professional education (e-learning) to online research of mental health. ${ }^{20}$

There are currently many different types of digital mental health treatment interventions for depression. ${ }^{1621}$ Review results have shown that computerised cognitivebehavioural therapy, which is also known as internetbased cognitive-behavioural therapy, provides efficacious and cost-effective treatment for patients with depression and anxiety. ${ }^{1722} 23$ Digital health interventions have many advantages for both patients and primary care professionals, as they can improve and support the treatment of mental disorders ${ }^{1724}$ by, for example, compensating for a shortage of mental health professionals. A potentially large number of patients can benefit from low-threshold access to digital mental health interventions, ${ }^{25}$ and these interventions may supplement and enrich standard care for this group of patients. ${ }^{16}$

Patients with mental disorders can still be treated by primary care professionals depending on their specific needs. But digital interventions have the advantage that patients can use them in their own time, according to their own needs and from any location. This is an important aspect for patients living in rural regions. ${ }^{25}$

Limited research exists into attitudes towards the use of digital treatment for mental disorders, ${ }^{24}{ }^{26}$ and the studies that do exist have investigated, for example, attitudes and patient satisfaction, statistical effects and barriers to the use, application and implementation of different types of digital therapeutic treatment options in a number of settings. ${ }^{26-29}$ However, they have not focused specifically on the primary care setting, or the perspectives of primary care professionals. To the best of our knowledge, this systematic review will be the first to report on primary care professionals' attitudes towards digital mental health interventions, whereby particular importance will be attached to investigating whether primary care professionals consider this kind of treatment for patients with mental disorders to be appropriate in view of the special role that they will play in the treatment of the patients. In view of the effective implementation and feasibility of the treatment, it is necessary to assess primary care professionals' acceptance of digital tools and their willingness to employ them.

\section{Research objective}

This systematic review will be conducted to explore current research findings into what motivates primary care professionals to use or not to use digital interventions in their daily work. The findings will also provide information on whether primary care professionals have an open mind with regard to digitalisation and the implementation of innovative digital technology in the treatment of their patients. This paper describes the study protocol of a mixed methods systematic review to explore the attitudes of primary care professionals towards the use of digital health interventions in the treatment of patients with mental disorders.

\section{METHODS}

This protocol conforms to the Preferred Reporting Items for Systematic Review and Meta-Analysis Protocols (PRISMA-P) checklist. $^{30}$ In the development of the mixed methods systematic review we will follow the PRISMA-P checklist.

\section{Eligibility criteria}

We will use a mixed methods systematic review to collect the findings from all published studies that are relevant to our research aim. We will systematically summarise the results in a single study on the attitudes of primary care professionals towards digital health interventions for the treatment of mental disorders in primary care. For this purpose, all findings will be collected, regardless of which of the three designs is employed, and combined and analysed in a single study on attitudes towards this specific intervention.

This review includes studies based on quantitative, qualitative and mixed methods research data in order 
to maximise the number of identified studies and findings. ${ }^{31}$ We included all studies published in English, German, Spanish, Russian, French and Dutch after 2010 and excluded reviews, opinion papers, editorials, study protocols, conference abstracts, commentaries and grey literature. E-health technology has improved dramatically in recent years. As this has obviously influenced users' attitudes, we decided only to include studies conducted in the past 10 years. The included studies had to include data on digital mental health interventions accessed via computer and/or mobile devices and under the guidance of professionals working in a primary care setting. These can include digital mental health interventions that GPs have recommended to their patients. After receiving instruction from their primary care professionals, patients are able to use the digital interventions independently. We will include all studies describing interventions aimed at treating depression, mood disorders and anxiety. We defined the primary care setting as an ambulatory healthcare facility in which primary care professionals such as GPs and nurses work. For example, we will exclude studies involving digital interventions delivered by psychotherapists in an inpatient setting. We will also exclude interventions involving only video consultations to replace regular face-to-face consultations.

We will identify attitudes such as acceptance, experience, views and the perspectives of primary care professionals towards digital mental health interventions for the treatment of patients with mental disorders such as depression, mood disorders and anxiety. In addition, facilitators and barriers to the willingness to apply the interventions, and views on the feasibility of using them will also be identified. Table 1 offers an overview of inclusion and exclusion criteria.

\section{Information sources}

We searched MEDLINE via Ovid, Embase, CINAHL, PsycINFO via Ovid and Web of Science Core Collection. Two researchers (JH, MH) conducted the search in July 2020.

Our search strategy was based on database-specific controlled vocabulary, keywords and unspecified search terms. The search terms were identified on the basis of eligible studies identified by an exploratory search, expert consultation and the authors' expertise. The MeSH Browser ${ }^{32}$ and controlled vocabulary thesaurus were also used. To ensure the search strategy was accurate, we also used Peer Review of Electronic Search Strategy for systematic reviews. ${ }^{28}$ The search strategy was adapted for each database.

To identify further studies and grey literature, we also plan to perform backward and forward citation tracking of included studies using Scopus, as well as a free web search using Google Scholar.

\section{Search strategy}

The complete MEDLINE via Ovid search strategy as an example is shown in table 2 .

\section{Study records}

Data management

We will import all references from the five bibliographic databases into the reference management software $\mathrm{Citavi}^{33}$ and then remove duplicates. We will upload all included articles using the web app Rayyan ${ }^{34}$ for the screening process.

\begin{tabular}{lll}
\hline \multicolumn{2}{l}{ Table 1} & Inclusion and exclusion criteria \\
\hline Domain & Inclusion criteria & Exclusion criteria \\
\hline Study design & $\begin{array}{l}\text { Primary quantitative, qualitative and mixed methods } \\
\text { studies }\end{array}$ & Reviews \\
Type of publication & Academic journal publications & Opinion papers \\
& & Editorials \\
& & Study protocols \\
& & Conference abstracts \\
& & Commentaries \\
& & Grey literature
\end{tabular}

\begin{tabular}{lll} 
Intervention & $\begin{array}{l}\text { Interventions that treat mental disorders and include a } \\
\text { digital health intervention accessed via computer and/ } \\
\text { or mobile devices (ie, guided computerised cognitive- } \\
\text { behavioural therapy or online self-help programme) }\end{array}$ & $\begin{array}{l}\text { Other interventions (ie, only video consultation) } \\
\text { or video consultations delivered by } \\
\text { psychotherapy }\end{array}$ \\
\hline Health context & $\begin{array}{l}\text { Studies reporting on inventions aimed at treating } \\
\text { depression, mood disorders and anxiety, with no } \\
\text { restrictions on the age of study participants, sample size } \\
\text { and duration of the intervention }\end{array}$ & $\begin{array}{l}\text { Studies including patients with schizophrenia } \\
\text { and other psychoses, dementia, autism }\end{array}$ \\
& $\begin{array}{l}\text { Studies should assess primary care professionals' } \\
\text { eating disorders and developmental disorders }\end{array}$ & Other populations and setting \\
Population and & $\begin{array}{l}\text { attitudes (this includes regular primary care in which } \\
\text { patients are treated in an outpatient setting) }\end{array}$ & \\
\hline
\end{tabular}

Publication language Studies published in English, German, Spanish, Russian, and year $\quad$ French and Dutch since 2010 


\section{Table 2 MEDLINE search strategy (10 July 2020)

No Search strings \\ 1 ("FAMILY MEDICINE" or "FAMILY PRACTICE" or "GENERAL MEDICINE" or "GENERAL PRACTICE" or "PRIMARY CARE" or "PRIMARY HEALTH CARE" or "PRIMARY HEALTHCARE" or "PRIMARY HEALTH-CARE" or PRIMARYCARE or "PRIMARY- CARE").ti,ab. or exp GENERAL PRACTICE/ or exp PRIMARY HEALTH CARE/ or exp COMMUNITY HEALTH SERVICES/ or exp AMBULATORY CARE FACILITIES/ or exp COMPREHENSIVE HEALTH CARE/ or exp PRIMARY CARE NURSING/ \\ 2 (DOCTOR* or PHYSICIAN* or "FAMILY PRACTITIONER" or "GENERAL PRACTITIONE*" or "HEALTH CARE PROFESSIONAL" or "HEALTH CARE PROVIDER*" or "HEALTH CARE WORKER"” or "HEALTH PROFESSIONAL*" or "HEALTH CARE PERSONNEL"” or "HEALTH PERSONNEL" or "HEALTH PROVIDER*" or "HEALTH WORKER*" or "HEALTHCARE PROFESSIONAL"” or "HEALTHCARE PROVIDER*" or "HEALTHCARE WORKER*" or "HEALTHCARE PERSONNEL" or "HEALTH-CARE PROFESSIONAL"” or "HEALTH-CARE PROVIDER"” or "HEALTH-CARE WORKER" or "HEALTH-CARE PERSONNEL" or NURS* or "PRIMARY CARE PERSONNEL" or "PRIMARY CARE PROFESSIONAL"” or "PRIMARY CARE PROVIDER" or "PRIMARY CARE WORKER" or "PRIMARY HEALTH CARE PERSONNEL" or "PRIMARY HEALTH CARE PROFESSIONAL*" or "PRIMARY HEALTH CARE PROVIDER*" or "PRIMARY HEALTH CARE WORKER*" or "PRIMARY HEALTHCARE PERSONNEL" or "PRIMARY HEALTH-CARE PERSONNEL" or "PRIMARY HEALTHCARE PROFESSIONAL"” or "PRIMARY HEALTH-CARE PROFESSIONAL*" or "PRIMARY HEALTHCARE PROVIDER"” or "PRIMARY HEALTH-CARE PROVIDER" or "PRIMARY HEALTHCARE WORKER" or "PRIMARY HEALTH-CARE WORKER" or "PRIMARYCARE PERSONNEL" or "PRIMARY-CARE PERSONNEL" or "PRIMARYCARE PROFESSIONAL" or "PRIMARY- CARE PROFESSIONAL" or "PRIMARYCARE PROVIDER" or "PRIMARY-CARE PROVIDER" or "PRIMARYCARE WORKER*” or "PRIMARY-CARE WORKER*").ti,ab. or exp HEALTH PERSONNEL/}

3 (STRESS or "ADJUSTMENT DISORDER*" or AGORAPHOBIA or ANXIETY or BURNOUT or DEPRESS* or MENTAL* or "MOOD DISORDER" or "PANIC DISORDER"” or "PSYCHIATRIC DISORDER" or "SOCIAL ANXIETY").ti,ab. or exp MENTAL DISORDERS/ or exp DEPRESSIVE DISORDER/ or exp MENTAL HEALTH/ or exp MENTALLY ILL PERSONS/ or exp PSYCHIATRY/

4 (ANDROID or APP or APPS or "ARTIFICIAL COMPANION"” or "AUGMENTED REALIT" or "BLENDED TREATMENT"” or "CELL PHONE" or CELLPHONE* or CHAT* or CHATBOT* or "COMPANION ROBOT" or COMPUTER* or DIGITAL or "E HEALTH" or EHEALTH or "E-HEALTH" or EMAIL* or "E-MAIL" or "E-MENTAL HEALTH" or EXERGAME or EXERGAMES or EXERGAMING or GAME or GAMES or GAMIFICATION or GAMING or IPHONE* or "I-PHONE" or IPOD* or "I-POD"” or "MENTAL EHEALTH" or "MENTAL E-HEALTH" or MHEALTH or "M-HEALTH" or "MOBILE DEVICE*" or "MOBILE HEALTH" or "MOBILE-HEALTH" or "NINTENDO WII" or ONLINE* or PHONE* or REMOTE or ROBOT or ROBOTS or ROBOTIC or ROBOTICS or ROBOTTHERAPY or ROBOTTHERAPIES or "SERIOUS GAME" or SKYPE or "SMART PHONE" or SMARTPHONE* or SMS or "SOCIAL BOT" or TABLET* or TECHNOLOG* or "TELE HOME CARE" or "TELE MEDICINE" or TELECARE or "TELE-CARE" or TELECARING or "TELE-CARING" or TELECOMMUNICATION or TELECONSULTATION" or "TELE-CONSULTATION" or TELEHEALTH or "TELE-HEALTH" or TELEMEDICINE or TELEMENTAL or "TELE-MENTAL" or TELEMONITORING or "TELE-MONITORING" or TELENURSE or TELENURSING or TELEPHONE* or TELEREHABILITATION or "TELE-REHABILITATION" or TELEROBOT or TELEROBOTS or "TELE-ROBOT" or "TELE-ROBOTS" or TELEROBOTIC or TELEROBOTICS or TELETHERAPY or "TELE-THERAPY" or VIDEOCONFERENCE* or "VIRTUAL REALIT*" or "WEB BASED" or WEBBASED or "WEB-BASED" or WII).ti,ab. or exp TELENURSING/ or exp TELEMEDICINE/ or exp TECHNOLOGY/ or exp VIRTUAL REALITY/ or exp TELECOMMUNICATIONS/ or exp VIDEOCONFERENCING/ or exp TELEREHABILITATION/ or exp ROBOTICS/

\begin{tabular}{|c|c|}
\hline 5 & $\begin{array}{l}\text { (ACCEPT* or ADOPTION* or ATTITUDE* or AWARENESS or BARRIER* or EXPECTATION* or EXPERIENCE* or FACILITATOR* } \\
\text { or FEASIBILITY or OPINION }{ }^{*} \text { or PERCEPTION }{ }^{*} \text { or PERSPECTIVE* or "POINT OF VIEW" or POSITION* or RECOMMEND* or } \\
\text { REQU }^{*} \text { or SUGGEST }{ }^{*} \text { or USABILIT* or VIEW*).ti,ab. or exp ATTITUDE OF HEALTH PERSONNEL/ or exp AWARENESS/ }\end{array}$ \\
\hline 6 & 1 and 2 and 3 and 4 and 5 \\
\hline
\end{tabular}

\section{Selection process}

Two reviewers (MH, MvdA) will independently screen titles and abstracts first, and then screen the full texts of potentially relevant articles for eligibility. During the screening process, each study will be labelled 'include' or 'exclude'. If the reviewers cannot reach consensus at each step of the study selection process, the decision will be resolved through discussion or by consulting a third researcher.

In the final publication, we will use a PRISMA flow diagram ${ }^{30}$ to illustrate the study selection process. At the same time, we will also report reasons for the exclusion of full texts.

\section{Data collection process}

We will use a standardised data extraction sheet that was independently developed and piloted by two reviewers $(\mathrm{MH}$,
MvdA). In case a consensus on data extraction cannot be reached, a third researcher will be consulted. We will extract quantitative data from quantitative and mixed methods (only the quantitative components) studies and qualitative data from qualitative and mixed methods (only the qualitative components) studies. ${ }^{35}$

\section{Data items}

The following study characteristics will be extracted: reference (author(s) name, publication year, language, title), study design, study aim, country, funding, characteristics of study participants (kind of healthcare professional, sample size, age, sex), characteristics of the intervention (ie, type of intervention, characteristics of the sample, type of technology, duration of intervention), data collection assessment, 
review-specific results (ie, reported attitudes towards the intervention, experience/knowledge of digital health treatments).

\section{Risk of bias in individual studies}

We will use the Mixed Methods Appraisal Tool (MMAT), version $2018,{ }^{36}$ to critically appraise the quality of study methodologies and the designs of each included study. Poor quality studies will not be excluded. The MMAT will help us identify digital interventions that have been tested or successfully used and implemented in an ambulatory primary care setting. The MMAT will be conducted by two independent researchers.

\section{Data synthesis}

In this mixed methods systematic review, we will use the general 'Integrated methodology' framework described by Sandelowski $e t a l^{37}$ to synthesise the data. We will also base data synthesis on the Joanna Briggs Institute Reviewers' Manual 2014. ${ }^{31}$ Using this method, qualitative and quantitative data will be synthesised for use in a single mixed methods approach. ${ }^{38}$ A primary condition for the use of this framework is that both data findings address the same research question. ${ }^{39}$ A Bayesian approach will therefore be used to convert raw quantitative findings into qualitative themes or subthemes in order to produce a single overarching synthesis. ${ }^{31}$ Themes identified from qualitative data will be developed, and values from quantitative data will be codified to ensure compatibility (metaaggregative analysis).

We will use 'textual descriptions ${ }^{40}$ to 'translate' quantitative data ('qualitized data'). Textual descriptions of synthesised quantitative findings will be combined with synthesised findings based on qualitative data and integrated via a single mixed methods synthesis. Identifying and textualising themes from quantitative data is less error prone than quantifying qualitative data. ${ }^{38}$ Our aim is to achieve an overall configured analysis based on both data. The 'integrated design' will enable us to recognise whether findings confirm or refute each other, whereby our aim is to transform findings in such a way that they can be compared. ${ }^{37}$ The results will be presented and summarised as recommendations and conclusions in narrative form. We will report all significant and non-significant outcomes. Furthermore, we will conduct descriptive statistics data on the number and year of publication, country of publication, type of interventions/technology, kind of primary care professionals and mental health diagnosis. We will also present methodological details of the included studies that are based on the risk of bias appraisal tool.

Data extraction will be conducted by combining deductive and inductive approaches ${ }^{41}$ to develop categories and subcategories. First of all, we will rely on two separate frameworks and their constructs: 'Barriers and Facilitators to the implementation of shared decision making, ${ }^{42}$ and 'Unified Theory of Acceptance of Use of Technology' ${ }^{43}$ More specifically, we will focus on the following categories: barriers and facilitators, performance expectancy, effort expectancy, social influence and facilitating conditions, whereby barriers and facilitators will be further broken down into knowledge, attitudes and behaviours. Subsequently, we will use an inductive approach that is based on the data extracted from the included studies. Textual descriptions of quantitative data and text passages from qualitative studies will be assigned to these categories. This process will also enable us to develop new categories and subcategories. In the data extraction process, categories will be rendered more specific through differentiation and refinement.

Data extraction and the assignment of data to categories will be conducted by two independent researchers. If consensus cannot be reached, a third researcher will be consulted. In this way, it will be possible to ensure valid and reliable interpretation during the codification and categorisation processes.

\section{Potential practical implications}

The results of our review will provide a better understanding of the general implementation and application of digital mental health interventions and show how primary care professionals can improve the treatment of mental disorders in everyday practice. The results on facilitators and barriers, for example, will enable the way digital mental health interventions are delivered and their effectiveness to be improved.

\section{Patient and public involvement}

There was no direct patient and public involvement in the development of this protocol for a mixed methods review.

\section{ETHICS AND DISSEMINATION}

Ethical approval is not required. We aim to publish the results of the review in a peer-reviewed journal. Additionally, the review results will be disseminated in the German Society of General Practice and Family Medicine newsletter and at relevant scientific conferences.

\section{Author affiliations \\ ${ }^{1}$ Institute of General Practice, University of Frankfurt, Frankfurt am Main, Germany ${ }^{2}$ Center for Dementia Care, Institute of Applied Nursing Sciences, Department of Health, University of Applied Sciences of Eastern Switzerland, St Gallen, Switzerland ${ }^{3}$ International Graduate Academy, Institute for Health and Nursing Science, Medical Faculty, Martin Luther University Halle-Wittenberg, Halle (Saale), Germany ${ }^{4}$ Department of Family Medicine, Care and Public Health Research Institute (CAPHRI), Maastricht University, Maastricht, Netherlands \\ ${ }^{5}$ Department of Public Health and Primary Care, Katholieke Universiteit Leuven, Academic Centre for General Practice, Leuven, Belgium}

Acknowledgements The authors would like to thank Phillip Elliott for the final proofreading.

Contributors $\mathrm{MH}$ and MvdA were involved in developing the idea for this review. All authors made substantial contributions to the conception and design of the work. JH and MH developed the search strategy and conducted the search in July 2020. MH drafted the manuscript, and all authors reviewed it critically for important intellectual content. All authors gave their final approval to the manuscript version to be published and agreed to be accountable for all aspects of the manuscript. 
Funding The authors have not declared a specific grant for this research from any funding agency in the public, commercial or not-for-profit sectors.

Competing interests None declared.

Patient and public involvement Patients and/or the public were not involved in the design, or conduct, or reporting, or dissemination plans of this research.

Patient consent for publication Not required.

Provenance and peer review Not commissioned; externally peer reviewed.

Open access This is an open access article distributed in accordance with the Creative Commons Attribution Non Commercial (CC BY-NC 4.0) license, which permits others to distribute, remix, adapt, build upon this work non-commercially, and license their derivative works on different terms, provided the original work is properly cited, appropriate credit is given, any changes made indicated, and the use is non-commercial. See: http://creativecommons.org/licenses/by-nc/4.0/.

ORCID iD

Marjan van den Akker http://orcid.org/0000-0002-1022-8637

\section{REFERENCES}

1 WHO. Mental disorders: key facts, 2019. Available: https://www.who. int/news-room/fact-sheets/detail/mental-disorders [Accessed 2 Jul 2020].

2 James SL, Abate D, Abate $\mathrm{KH}$, et al. Global, regional, and national incidence, prevalence, and years lived with disability for 354 diseases and injuries for 195 countries and territories, 1990-2017: a systematic analysis for the global burden of disease study 2017 . The Lancet 2018;392:1789-858.

3 Whiteford HA, Ferrari AJ, Degenhardt L, et al. The global burden of mental, neurological and substance use disorders: an analysis from the global burden of disease study 2010. PLoS One 2015;10:e0116820.

4 Hawton K, Casañas I Comabella C, Haw C, et al. Risk factors for suicide in individuals with depression: a systematic review. J Affect Disord 2013;147:17-28.

5 Alexander C, Fraser J. General practitioners' management of patients with mental health conditions: the views of general practitioners working in rural north-western New South Wales. Aust J Rural Health 2008;16:363-9.

6 von GC, Checchia C, Hausen A. Depressionsbehandlung AUS hausärztlicher Sicht: Eine qualitative analyse von Experteninterviews. 96. Deutscher Ärzteverlag | ZFA | Zeitschrift für Allgemeinmedizin 2020.

7 Anthony JS, Baik S-Y, Bowers BJ, et al. Conditions that influence a primary care clinician's decision to refer patients for depression care. Rehabil Nurs 2010;35:113-22.

8 Jacobi F, Höfler M, Siegert J, et al. Twelve-month prevalence, comorbidity and correlates of mental disorders in Germany: the mental health module of the German health interview and examination survey for adults (DEGS1-MH). Int J Methods Psychiatr Res 2014;23:304-19.

9 Trautmann S, Beesdo-Baum K. The treatment of depression in primary care. Deutsches Ärzteblatt international 2017;117:721-8.

10 Caldwell TM, Caldwell TM, Jorm AF, Knox S, et al. General practice encounters for psychological problems in rural, remote and metropolitan areas in Australia. Aust N Z J Psychiatry 2004;38:774-80.

11 Dijksman I, Dinant G-J, Spigt MG. eDiagnostics: a promising step towards primary mental health care. Fam Pract 2013;30:695-704

12 Thabrew H, Stasiak K, Hetrick SE, et al. E-Health interventions for anxiety and depression in children and adolescents with long-term physical conditions. Cochrane Database Syst Rev 2018;8:CD012489.

13 Moessner M, Bauer S. E-Mental-Health und internetbasierte Psychotherapie. Psychotherapeut 2017;62:251-66.

14 Rai M, Vigod SN, Hensel JM. Barriers to office-based mental health care and interest in E-Communication with providers: a survey study. JMIR Ment Health 2016;3:e35.

15 Kruse RL, Koopman RJ, Wakefield BJ. Internet use by primary care patients: where is the digital divide? Fam Med 2012;44:342-7.

16 Zwerenz R, Beutel ME. Online-Intervention Zur Behandlung von psychsichen Erkrankungen und Belastungen: Welche aktuellen Ansätze gibt ES und wie wirksam sind diese? Arbeitsmed Sozialmed Umweltmed 2017;52:452-60.

17 Reynolds J, Griffiths KM, Cunningham JA, et al. Clinical practice models for the use of E-Mental health resources in primary health care by health professionals and peer workers: a conceptual framework. JMIR Ment Health 2015;2:e6.

18 Andrews G, Cuijpers P, Craske MG, et al. Computer therapy for the anxiety and depressive disorders is effective, acceptable and practical health care: a meta-analysis. PLoS One 2010;5:e13196.

19 Klein JP, Berger T. Internetbasierte psychologische Behandlung bei Depressionen. Verhaltenstherapie 2013;23:149-59.

20 Riper $\mathrm{H}$, Andersson $\mathrm{G}$, Christensen $\mathrm{H}$, et al. Theme issue on e-mental health: a growing field in Internet research. J Med Internet Res 2010;12:e74.

21 Renton T, Tang H, Ennis N, et al. Web-Based intervention programs for depression: a scoping review and evaluation. J Med Internet Res 2014;16:e209.

22 Kaltenthaler E, Shackley $\mathrm{P}$, Stevens $\mathrm{K}$, et al. A systematic review and economic evaluation of computerised cognitive behaviour therapy for depression and anxiety. Health Technol Assess 2002;6:1-89 https:// www.ncbi.nlm.nih.gov/books/NBK69452/

23 Spek V, Cuijpers P, Nyklícek I, et al. Internet-Based cognitive behaviour therapy for symptoms of depression and anxiety: a metaanalysis. Psychol Med 2007;37:319-28.

24 Breedvelt JJ, Zamperoni V, Kessler D, et al. Gps' attitudes towards digital technologies for depression: an online survey in primary care. Br J Gen Pract 2019;69:e164-70.

25 Müller-Mielitz S, Lux T. E-Mental-Health - am Beispiel von internetbasierten Gesundheitsinterventionen. Wiesbaden: Springer Fachmedien Wiesbaden, 2017.

26 Topooco N, Riper H, Araya R, et al. Attitudes towards digital treatment for depression: a European stakeholder survey. Internet Interv 2017:8:1-9.

27 Meyer B, Bierbrodt J, Schröder J, et al. Effects of an Internet intervention (Deprexis) on severe depression symptoms: randomized controlled trial. Internet Interv 2015;2:48-59.

28 McGowan J, Sampson M, Salzwedel DM, et al. PRESS Peer Review of Electronic Search Strategies: 2015 Guideline Statement. J Clin Epidemiol 2016;75:40-6.

29 Richards D, Richardson T. Computer-Based psychological treatments for depression: a systematic review and meta-analysis. Clin Psychol Rev 2012;32:329-42.

30 Liberati A, Altman DG, Tetzlaff J, et al. The PRISMA statement for reporting systematic reviews and meta-analyses of studies that evaluate health care interventions: explanation and elaboration. PLoS Med 2009;6:e1000100.

31 The Joanna Briggs Institute. The Joanna Briggs Institute Reviewers' Manual 2014: Methodology for JBI Mixed Methods Systematic Reviews 2014

32 U.S. National library of medicine. Medical subject Headings 2020, 2020. Available: https://meshb.nlm.nih.gov/search

33 Swiss academic software. Citavi, 2020. Available: https://www.citavi com/de [Accessed 30 Jun 2020].

34 Ouzzani M, Hammady H, Fedorowicz Z, et al. Rayyan-a web and mobile APP for systematic reviews 2016.

35 Aromataris E, Munn Z. JBI manual for evidence synthesis, 2020. Available: https://wiki.jbi.global/display/MANUAL [Accessed $23 \mathrm{Ju}$ 2020].

36 Hong QN, Pluye P, bregues S F, et al. Mixed methods appraisal tool (MMAT), version 2018: user guide. McGill University Department of Family Medicine, Canadian, 2018.

37 Sandelowski M, Voils Cl, Barroso J. Defining and designing mixed research synthesis studies. Res Sch 2006;13:29.

38 Pearson A, White H, Bath-Hextall F, et al. A mixed-methods approach to systematic reviews. Int J Evid Based Healthc 2015;13:121-31.

39 Noyes J, Booth A, Moore G, et al. Synthesising quantitative and qualitative evidence to inform guidelines on complex interventions: Clarifying the purposes, designs and outlining some methods. $B M$ Glob Health 2019;4:e000893.

40 Sandelowski M, Leeman J, Knafl K, et al. Text-in-context: a method for extracting findings in mixed-methods mixed research synthesis studies. J Adv Nurs 2013;69:1428-37.

41 Kuckartz U. Mixed methods: Methodologie, Forschungsdesigns und Analyseverfahren. Wiesbaden: Springer Fachmedien Wiesbaden, 2014

42 Légaré F, Ratté S, Gravel K, et al. Barriers and facilitators to implementing shared decision-making in clinical practice: update of a systematic review of health professionals' perceptions. Patient Educ Couns 2008;73:526-35.

43 Venkatesh V, Morris MG, Davis GB. User acceptance of information technology: toward a unified view. MIS Quarterly 2003;27:425-78. 